\title{
Risk of Venous Thromboembolism After Air Travel
}

\author{
Interaction With Thrombophilia and Oral Contraceptives
}

Ida Martinelli, MD, PhD; Emanuela Taioli, MD, PhD; Tullia Battaglioli, MD; Gian Marco Podda, MD; Serena Maria Passamonti, MD; Paola Pedotti, BSc; Pier Mannuccio Mannucci, MD

\begin{abstract}
Background: Conflicting data are available on air travel as a risk factor for venous thromboembolism. To our knowledge, there are no studies investigating whether individuals with thrombophilia and those taking oral contraceptives are more likely to develop venous thromboembolism during flights than those without these risk factors.
\end{abstract}

Participants and Methods: The study sample consisted of 210 patients with venous thromboembolism and 210 healthy controls. DNA analysis for mutations in factor $\mathrm{V}$ and prothrombin genes and plasma measurements of antithrombin, protein $\mathrm{C}$, protein $\mathrm{S}$, total homocysteine levels, and antiphsopholipid antibodies were performed.

Results: In the month preceding thrombosis for patients, or the visit for controls, air travel was reported by 31 patients $(15 \%)$ and 16 controls (8\%), with an oddsratio of 2.1 (95\% confidence interval, 1.1-4.0).
Thrombophilia was present in 102 patients (49\%) and 26 controls (12\%), and oral contraceptives were used by 48 patients and 19 controls $(61 \%$ and $27 \%$ of those of reproductive age, respectively). After stratification for the presence of air travel and thrombophilia, the odds ratio for thrombosis in individuals with both risk factors was 16.1 (95\% confidence interval, 3.6-70.9). Stratification for the presence of air travel and oral contraceptive use gave an odds ratio of 13.9 (95\% confidence interval, 1.7$117.5)$ in women with both risk factors.

Conclusions: Air travel is a mild risk factor for venous thromboembolism, doubling the risk of the disease. When thrombophilia or oral contraceptive use is present, the risk increases to 16-fold and 14-fold, respectively, indicating a multiplicative interaction.

Arch Intern Med. 2003;163:2771-2774
From the Angelo Bianchi Bonomi Hemophilia and Thrombosis Center (Drs Martinelli, Battaglioli, Podda, Passamonti, and Mannucci) and Molecular and Genetic Epidemiology Unit (Dr Taioli and Ms Pedotti), Istituto di Ricovero e Cura a Carattere Scientifico, Maggiore Hospital, University of Milan, Milan, Italy. The authors have no relevant financial interest in this article.

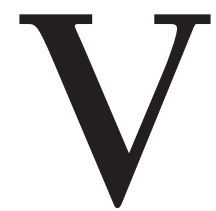

ENOUS THROMBOEMBOLISM (VTE) is a multifactorial disease resulting from the interaction between genetic and environmental risk factors. ${ }^{1}$ The former includes abnormalities causing inherited thrombophilia, such as deficiencies of the naturally occurring anticoagulants antithrombin, protein $\mathrm{C}$, protein S, and the gain-of-function mutations in genes encoding coagulation factor V (factor V Leiden) and prothrombin. The environmental, transient risk factors associated with an increased risk of VTE are cancer, recent surgery, pregnancy and puerperium, use of oral contraceptives, and prolonged immobilization.

Recently, it has been suggested that air travel should be included among transient risk factors for VTE. The first observation that air or surface travel might be associated with VTE was made in the mid $1960 \mathrm{~s}^{2}$ and was recently confirmed in 2 case-control studies. ${ }^{3,4}$ However, another case-control study limited to air travel failed to confirm such an association. ${ }^{5}$ After the recent demonstration of an association between air travel and symptomatic pulmonary embolism (PE) ${ }^{6}$ simple behavioral measures were rec- ommended during flights, such as increased water consumption, limitation of alcohol, avoidance of constricting clothes and leg crossing, and mild leg exercise. It has also been pointed out that more aggressive prophylactic measures, such as elastic stockings or anticoagulant drugs, should be implemented in high-risk individuals, such as those with thrombophilia. ${ }^{7}$ Although this recommendation is clinically meaningful, there is no evidence that the risk of VTE after air travel is influenced by the presence of thrombophilia. To establish whether air travel is a risk factor for VTE, and to investigate the

\section{See also pages 2674 , 2759, and 2766}

interaction between air travel and thrombophilia, we carried out a case-control study in which the potential for bias was minimized. We also evaluated the interaction between air travel and oral contraceptive use, which is a common and independent risk factor for VTE. Our study could help to determine whether antithrombotic prophylaxis should be given during air travel to individuals at a potentially high risk of VTE. 
Table 1. General Characteristics of the Study Population

\begin{tabular}{lcc} 
Characteristic & $\begin{array}{c}\text { Patients } \\
(\mathbf{n = 2 1 0 )}\end{array}$ & $\begin{array}{c}\text { Controls } \\
(\mathbf{n}=\mathbf{2 1 0})\end{array}$ \\
\hline Sex, M/F, No. & $107 / 103$ & $115 / 95$ \\
Age, median (range), y & $42(20-71)$ & $43(18-69)$ \\
Education level, No. & & \\
$\quad$ Grade school & 17 & 18 \\
$\quad$ High school & 56 & 56 \\
$\quad$ College & 95 & 94 \\
$\quad$ University & 42 & 42 \\
Body mass index, mean $\pm \mathrm{SD}, \mathrm{kg} / \mathrm{m}^{2}$ & $25.3 \pm 4.5$ & $24.0 \pm 3.7$ \\
\end{tabular}

METHODS

PATIENTS

Consecutive patients attending the Thrombosis Center from January 1, 1999, to September 30, 2001, for a thrombophilia screening were included in the study if they had a first and documented episode of proximal deep vein thrombosis (DVT) and/or $\mathrm{PE}$ in the last 24 months. Patients younger than 20 years or older than 70 years and those with known malignant diseases were excluded from the study. Proximal DVT was objectively diagnosed by (Doppler) ultrasound examination or venography and PE by perfusion or ventilation-perfusion lung scan, computed tomography, or angiography. At the time of the visit, patients were asked about the presence of transient risk factors in the month preceding the event, including air travel, oral contraceptive use or hormone replacement therapy, pregnancy, surgery, trauma, and immobilization. At this time, interviewing physicians were unaware of the presence of thrombophilia. When air travel was reported, the distance and duration of the flight and the class of travel were asked. Long-distance flights were considered those of at least 8 hours' duration, according to Scurr et al. ${ }^{8}$

\section{CONTROLS}

Control subjects were individuals who volunteered to be screened for thrombophilia in the same period of referral as patients. This group consisted of friends or partners of the entire population of patients referred to the Thrombosis Center, with the only exclusion criterion being a positive personal history for thrombosis and overt malignant diseases. A validated questionnaire was used to ascertain the absence of thrombosis in their life. ${ }^{9}$ One control was matched to each patient for age $( \pm 5$ years), sex, and education level (4 different levels from grade school to university). At the time of the visit, controls were asked about the presence in the preceding month of the same transient risk factors for thrombosis that were asked of patients.

All the study participants gave their written consent. The study protocol is consistent with the principle of the Declaration of Helsinki.

\section{LABORATORY TESTS}

Laboratory tests were carried out and interpreted by independent and blinded technicians. Thrombophilia screening included DNA analysis for the 1691 guanine to adenine substitution in coagulation factor V gene (factor V Leiden) and for the 20210 guanine to adenine substitution in the prothrombin gene; plasma levels of the naturally occurring anticoagulants antithrombin, protein $C$, and protein S; the presence of antiphospholipid antibodies (lupus anticoagulant and anticardiolipin antibodies); and plasma levels of fasting and post-methionine load total homocysteine.

Blood was collected into vacuum tubes containing 3.8\% (weight per volume) sodium citrate as anticoagulant for co- agulation tests and EDTA for homocysteine measurements. The DNA analysis was carried out as described by de Ronde and Bertina $^{10}$ for factor V Leiden and by Poort et $\mathrm{al}^{11}$ for the prothrombin mutation. Antithrombin, protein $C$, and protein $S$ deficiency were diagnosed on the basis of laboratory measurements as described elsewhere. ${ }^{12}$ Total plasma homocysteine was measured by high-performance liquid chromatography after overnight fasting and 4 hours after an oral methionine loading $\left(3.8 \mathrm{~g} / \mathrm{m}^{2}\right.$ of body surface area) as previously described..$^{13}$

All individuals included in the study underwent a complete thrombophilia screening, except for 60 patients in whom protein $C$ and protein $S$ levels could not be evaluated because of ongoing oral anticoagulant treatment at the time of blood sampling, since measurements of vitamin K-dependent proteins are unreliable during such therapy.

\section{STATISTICAL ANALYSIS}

On the assumption that 2 persons out of 10 fly and that the relative risk of VTE associated with air travel is 3, we calculated before starting the study that 100 patients with thrombosis and 100 controls would be necessary for an $80 \%$ power of the study. Odds ratios and $95 \%$ confidence intervals (CIs) were calculated to estimate the association between VTE and air travel. By means of a logistic regression model, odds ratios were adjusted for possible confounders, such as age, sex, and body mass index. The same model was used to evaluate the interaction between air travel and the presence of thrombophilia or the use of oral contraceptives. The analyses were performed using statistical software (SAS version 8.1; SAS Institute Inc, Cary, NC).

\section{RESULTS}

A total of 232 patients with a first episode of VTE were identified during the study period. Twenty-two of them were excluded because the objective documentation of VTE was inadequate or because of age limits. Therefore, 210 patients were included in the study, and the same number of controls were selected according to the matching criteria. One hundred seventy-two patients had proximal DVT of the lower limbs, complicated by symptomatic $P E$ in 39 . The remaining 38 patients had $P E$ with no diagnosed DVT. The median time elapsed from VTE to the visit was 5 months (range, 1-24 months).

Table 1 gives the general characteristics of the study population. In 100 patients (48\%) no transient risk factors for VTE were identified (Table 2). Air travel was reported by 31 patients (15\%) and 16 controls (8\%), with an odds ratio of 2.1 (95\% CI, 1.1-4.0). Long-distance flights were made by 11 patients ( 9 in economy class) and 4 controls ( 3 in economy class), for a mean duration of 13 hours 30 minutes and 19 hours, respectively. The odds ratio for VTE associated with long-distance flights was 3.0 (95\% CI, 0.9-9.5). Short-distance flights were made by the remaining 20 patients (16 in economy class) and 12 controls ( 9 in economy class), for a mean duration of 2 hours and 1 hour 30 minutes, respectively. Among transient risk factors for VTE, the most common was oral contraceptive use, both in patients and controls, being present in $61 \%$ and $27 \%$ of women of fertile age, respectively.

Table 2 also provides the type of thrombophilic abnormalities diagnosed in patients and controls. Thrombophilia was present in 102 patients (49\%) and 26 controls (12\%), with an odds ratio of 6.7 (95\% CI, 4.1-10.9). Overall, fac- 
tor V Leiden was found in 40 patients (19\%) and 8 controls (4\%), prothrombin mutation in 29 patients (14\%) and 6 controls (3\%), deficiency of anticoagulant proteins in 5 patients ( $2 \%$ ) ( 3 antithrombin, 1 protein $C$, and 1 protein Sdeficiency) and in none of the controls, antiphospholipid antibodies in 12 patients (6\%) and in none of the controls, and hyperhomocystinemia in 35 patients (17\%) and 15 controls (7\%). Excluding isolated hyperhomocystinemia, which is a metabolic abnormality, coagulation abnormalities were present in 72 patients (34\%) and 13 controls (6\%), with an odds ratio of 7.7 (95\% CI, 4.1-14.5). The distribution of thrombophilia was similar in patients who developed VTE after air travel and in those who did not fly before the event $(58 \%$ and $47 \%$, respectively), as well as the distribution of transient risk factors (oral contraceptive use, 67\% and 60\%; pregnancy or puerperium, $8 \%$ and $9 \%$; surgery, trauma, or prolonged immobilization, 29\% and 28\%, respectively).

The interaction between air travel and thrombophilia in determining VTE is given in Table 3. Considering as the reference group the individuals who did not fly and did not have thrombophilia, the risk of VTE was increased approximately 6-fold in the presence of thrombophilia, 2-fold in the presence of air travel, and 16-fold in the presence of both. When adjusted for age, sex, and body mass index, data did not substantially change.

Table 4 gives the interaction between air travel and oral contraceptive use. Compared with the reference group of women who did not fly or use oral contraceptives, the risk for VTE was approximately 4 times higher for oral contraceptive users, 2 times higher for those who flew, and 14 times higher when both risk factors were present. Adjustment for age and body mass index did not change the results.

\section{COMMENT}

This study demonstrates that air travel is a mild risk factor for VTE, being associated with a 2-fold increased risk. Approximately three fourths of the flights reported by patients and controls were short-distance flights (less than 8 hours) in economy class. The relative risk of VTE was slightly higher (3-fold) when only long-distance flights were considered. The risk was markedly increased when other common risk factors for VTE, such as thrombophilia and oral contraceptive intake, were concomitantly present. The approximately 16-fold and 14-fold increased risk of VTE for carriers of thrombophilia and users of oral contraceptives, respectively, indicates a multiplicative interaction between these risk factors and air travel.

To date, available studies on the association between air travel and VTE show conflicting results and have various drawbacks. The first 2 case-control studies ${ }^{3,4}$ that showed an association between travel and VTE did not differentiate air travel from surface travel and suffered from referral bias, and one of them had an inappropriately selected control group. ${ }^{3}$ Referral bias was avoided in another case-control study ${ }^{5}$ in which controls were individuals presenting with clinically suspected DVT that was confirmed or excluded by objective techniques. Despite the large sample size, this study ${ }^{5}$ failed to show an association between air travel and DVT. The limitation was that an unusually small number of individuals ( 4 cases [2\%] and 13 controls [2\%]) had been exposed to the risk factor under evaluation (ie, air travel, in

\begin{tabular}{|c|c|c|}
\hline Variable & Patients & Controls \\
\hline \multicolumn{3}{|l|}{ Thrombophilia, No. (\%) } \\
\hline Any type & $102(49)$ & $26(12)$ \\
\hline Factor V Leiden & $32(15)$ & $6(3)$ \\
\hline Prothrombin G20210A & $13(6)$ & $4(2)$ \\
\hline Antithrombin deficiency & $2(1)$ & 0 \\
\hline Protein C deficiency & 0 & 0 \\
\hline Protein S deficiency & 0 & 0 \\
\hline Antiphospholipid antibodies & $9(4)$ & 0 \\
\hline Hyperhomocysteinemia & $30(14)$ & $13(6)$ \\
\hline Combined abnormalities* & $16(8)$ & $3(1)$ \\
\hline \multicolumn{3}{|l|}{ Transient risk factors, No. (\%) } \\
\hline None & $100(48)$ & $202(96)$ \\
\hline Surgery & $26(12)$ & 0 \\
\hline Trauma/immobilization & $34(16)$ & 0 \\
\hline Oral contraceptives† & $48(61)$ & $19(27)$ \\
\hline Hormone replacement therapy & $8(33)$ & $9(37)$ \\
\hline Pregnancy/puerperium† & $7(8)$ & 0 \\
\hline
\end{tabular}

*In patients, there were 13 double abnormalities: 5 factor $V$ Leiden and prothrombin G20210A, 1 prothrombin G20210A and antithrombin deficiency, 1 prothrombin G20210A and protein C deficiency, 1 prothrombin G20210A and protein S deficiency, 2 prothrombin G20210A and antiphospholipid antibodies, and 3 prothrombin G20210A and hyperhomocysteinemia; and there were 3 triple abnormalities: 1 factor V Leiden, prothrombin G20210A, and antiphospholipid antibodies and 2 factor V Leiden, prothrombin G20210A, and hyperhomocysteinemia. In controls, there were 3 double abnormalities: 1 factor $\mathrm{V}$ Leiden and prothrombin G20210A, 1 factor V Leiden and hyperhomocysteinemia, and 1 prothrombin G20210A and hyperhomocysteinemia.

†Percentage calculated on the number of women of reproductive age.

$\ddagger$ Percentage calculated on the number of women not of reproductive age.

the month preceding the visit). Possible reasons might be the advanced mean age of that study population (63 years) and the inclusion of individuals with malignant disease. Although our control group was selected with different criteria, we are confident that it was appropriately chosen because thrombosis was ruled out by means of a structured validated questionnaire. ${ }^{9}$ In addition, we adopted strict matching criteria that make the chance of exposure to the risk factor under evaluation very similar between cases and controls. In fact, there is no reason to think that individuals of the same sex, age, and, above all, education level have different opportunities to travel by air. Moreover, unlike others, we chose not to include individuals with overt malignant disease or previous episodes of VTE, because these are 2 strong and independent risk factors for recurrence that may affect the results. Another important point is that all our patients with lower-limb DVT had a proximal location of thrombi, therefore avoiding the potential for inaccurate ultrasonographic assessment of distal thrombosis. This limitation is a major problem in the randomized trial by Scurr et al, ${ }^{8}$ in which individuals with DVT were all allocated to the group not wearing elastic stockings, and all had distal DVT diagnosed with an inadequate method, such as ultrasonography. ${ }^{14}$ Therefore, the possibility of falsepositive results cannot be definitely ruled out in that study.

This study has some limitations. The population of patients referred to the Thrombosis Center is highly selected, but exclusion of patients with recurrent VTE is likely to have limited the selection of the most severe cases. In addition, there is no reason to think that patients with a certain risk factor for VTE (for example, air travel or oral contracep- 
Table 3. Interaction Between Air Travel and the Presence of Thrombophilia in Determining Venous Thromboembolism

\begin{tabular}{cccccc}
\hline Air Travel & Thrombophilia & No. of Patients & No. of Controls & Odds Ratio (95\% Cl) & Adjusted Odds Ratio (95\% Cl)* \\
\hline No & No & 95 & 170 & 1 (Referent) & 1 (Referent) \\
No & Yes & 84 & 24 & $6.3(3.7-10.5)$ & $6.6(3.9-11.3)$ \\
Yes & No & 13 & 14 & $1.7(0.7-3.7)$ & $1.7(0.7-4.0)$ \\
Yes & Yes & 18 & $16.1(3.6-70.9)$ & $16.8(3.8-74.7)$
\end{tabular}

Abbreviation: $\mathrm{Cl}$, confidence interval.

*Adjusted for age, sex, and body mass index.

Table 4. Interaction Between Air Travel and the Use of Oral Contraceptives in Determining Venous Thromboembolism in 79 Patients and 71 Controls of Reproductive Age

\begin{tabular}{cccccc}
\hline Air Travel & Oral Contraceptives & No. of Patients & No. of Controls & Odds Ratio (95\% Cl) & Adjusted Odds Ratio (95\% CI)* \\
\hline No & No & 27 & 47 & 1 (Referent) & 1 (Referent) \\
No & Yes & 40 & 18 & $3.9(1.9-8.0)$ & $4.2(1.9-9.3)$ \\
Yes & No & 4 & 5 & $1.4(0.3-5.6)$ & $1.8(0.4-7.6)$ \\
Yes & Yes & 8 & 1 & $13.9(1.7-117.5)$ & $23.4(2.6-211.2)$
\end{tabular}

Abbreviation: $\mathrm{Cl}$, confidence interval.

*Adjusted for age and body mass index.

tive use) were preferentially referred to our center instead of others. Regarding ascertainment bias, we believe that this was limited by using the same questionnaire to interview both patients and controls, whereas the possibility of recall bias cannot be completely ruled out, especially for patients who were asked about risk factors for VTE in the month preceding the event at a median time of 5 months (range, 1-24 months) elapsed from the event to the visit.

Although this is the first study, to our knowledge, to evaluate the interaction between air travel and thrombophilia, it has been speculated that VTE after air travel is more likely to occur in individuals with thrombophilia. ${ }^{7,8,15} \mathrm{We}$ estimated the magnitude of the risk of VTE associated with air travel both in carriers of thrombophilia and in users of oral contraceptives, finding a strong association. The estimates of the risk of VTE after stratification according to the presence of air travel and thrombophilia or oral contraceptive use are statistically significant but rather unstable, as shown by the wide CIs. This is due to the small number of controls who reported recent air travel and had thrombophilia or used oral contraceptives. However, the lower limits of the CIs, nearly 4 for air travel plus thrombophilia and nearly 2 for air travel plus oral contraceptive use, indicate that individuals with 1 of these 2 risk factors who fly need more aggressive prevention measures than those without. Simple behavioral measures, such as adequate hydration and leg exercise, may not be sufficient to prevent VTE in individuals with thrombophilia or oral contraceptive users, even for short-distance flights. Only 1 trial $^{16}$ has been carried out to evaluate the effectiveness of prophylaxis in high-risk individuals, giving results in favor of a single dose of low-molecular-weight heparin instead of aspirin. Which kind of prophylaxis should be suggested before flying for individuals with thrombophilia and women taking oral contraceptives, either heparin or mechanical measures, such as elastic compression stockings, remains to be assessed by specifically tailored studies.

Accepted for publication December 6, 2002.
This study was supported by grant MM06157335 from the Ministry of University and Scientific and Technologic Research, Rome, Italy.

We thank Mihaela Savin for her assistance in preparing the manuscript.

Corresponding author: Ida Martinelli, MD, PhD, Hemophilia and Thrombosis Center, IRCCS Maggiore Hospital, University of Milano, Via Pace 9, 20122 Milano, Italy.

\section{REFERENCES}

1. Martinelli I. Risk factors in venous thromboembolism. Thromb Haemost. 2001; 86:395-403

2. Homans J. Thrombosis of the deep leg veins due to prolonged sitting. N Engl J Med. 1954;250:148-149.

3. Ferrari E, Chevallier T, Chapelier A, Baudouy M. Travel as a risk factor for venous thromboembolic disease: a case-control study. Chest. 1999;115:440-444.

4. Samama MM. An epidemiologic study of risk factors for deep vein thrombosis in medical outpatients: the Sirius Study. Arch Intern Med. 2000;160:3415-3420.

5. Kraaijenhagen RA, Haverkamp D, Koopman MMW, Prandoni P, Piovella F, Büller HR. Travel and risk of venous thrombosis. Lancet. 2000;356:1492.

6. Lapostolle F, Surget V, Borron SW, et al. Severe pulmonary embolism associated with air travel. N Engl J Med. 2001;345:779-829.

7. Hirsh J, O'Donnell M. Venous thromboembolism after long flights: are airlines to blame? Lancet. 2001;357:1461-1462.

8. Scurr JH, Machin SJ, Bailey-King S, Mackie IJ, McDonald S, Coleridge Smith PD. Frequency and prevention of symptomless deep-vein thrombosis in longhaul flights: a randomised trial. Lancet. 2001;357:1485-1489.

9. Frezzato M, Tosetto A, Rodeghiero F. Validated questionnaire for the identification of previous personal or familial venous thromboembolism. Am J Epidemiol. 1996;143:1257-1265.

10. de Ronde H, Bertina RM. Laboratory diagnosis of APC-resistance. Thromb Haemost. 1994;72:880-886.

11. Poort SR, Rosendaal FR, Reitsma PH, Bertina RM. A common genetic variation in the 3'-untranslated region of the prothrombin gene is associated with elevated plasma prothrombin levels and an increase in venous thrombosis. Blood. 1996:88:3698-3703

12. Martinelli I, Mannucci PM, De Stefano V, et al. Different risks of thrombosis in four coagulation defects associated with inherited thrombophilia: a study of 150 families. Blood. 1998;92:2353-2358.

13. Zighetti ML, Cattaneo M, Falcon CR, et al. Absence of hyperhomocysteinemia in ten patients with primary pulmonary hypertension. Thromb Res. 1997;85:279-282.

14. Kearon C, Ginsberg JS, Hirsh J. The role of venous ultrasonography in the diagnosis of suspected deep venous thrombosis and pulmonary embolism. Ann Intern Med. 1998:129:1044-1049.

15. Rege KP, Bevan DH, Chitolie A, Shannon MS. Risk factors and thrombosis after airline flight. Thromb Haemost. 1999;81:995-996.

16. Cesarone MR, Belcaro G. Nicolaides AN, et al. Venous thrombosis from air travel: the LONFLIT3 Study: prevention with aspirin vs low-molecular-weight heparin (LMWH) in high-risk subjects: a randomized trial. Angiology. 2002;53:1-6. 